\title{
Strategie di consumer-brand engagement. Il punto di vista delle imprese e delle agenzie di comunicazione
}

\author{
Maria Vernuccio - Gabriele Qualizza - Andrea Buratti \\ Federica Ceccotti
}

\begin{abstract}
Obiettivi del paper: Il lavoro si propone di studiare le strategie di consumerbrand engagement (CBE), sviluppando un originale framework integrato, basato sui punti di vista dei player appartenenti a due categorie chiave del communication network: le imprese inserzioniste ('clicks-and-mortar' e 'pure play') e le agenzie di comunicazione (tradizionali e digitali).

Metodologia: Seguendo un approccio esplorativo ed induttivo di tipo qualitativo abbiamo condotto 44 interviste in profondità a esperti di marketing e di comunicazione (consulenti e manager). I dati qualitativi sono stati analizzati attraverso unanalisi tematica del contenuto.

Risultati: I risultati mostrano similarità e specificità nelle prospettive manageriali rispetto al CBE in relazione alla categoria di appartenenza e allorientamento al digital degli intervistati.

Limiti della ricerca: Questo paper non è esente da limiti, soprattutto legati alla natura qualitativa ed esplorativa del lavoro. I risultati non possono essere generalizzati.

Implicazioni pratiche: I risultati offrono utili suggerimenti ai diversi attori studiati (imprese 'clicks-and-mortar' e 'pure play', agenzie tradizionali e digitali) per migliorare l'efficacia delle strategie di CBE.

Originalità del paper: Le precedenti ricerche sul CBE si sono concentrate soprattutto sull'analisi del punto di vista del consumatore. Focalizzando l'attenzione sui differenti tipi di imprese e agenzie, il nostro studio rappresenta il primo contributo accademico che mira ad approfondire il punto di vista manageriale all'interno di un framework concettuale che integra $i$ diversi temi chiave relativi allo sviluppo delle strategie di CBE.
\end{abstract}

Parole chiave: consumer-brand engagement; agenzia tradizionale e digitale; impresa 'clicks-and-mortar' e 'pure play'

Purpose of the paper: The aim of the paper is to deepen the managerial perspectives towards consumer-brand engagement strategies (CBE) by developing an original integrative framework based upon two key players' categories within the marketing communication network: enterprises ('clicks-and-mortar' and 'pure play') and agencies (traditional and digital).

Methodology: Following an exploratory inductive and qualitative approach, we have drawn upon 44 in-depth interviews with marketing and communication experts 
sinergie Vol. 36, N. 106, 2018

(consultants and managers). Qualitative data was analysed through a thematic content analysis.

Findings: The findings show similarities and specificities in the managerial perspectives towards CBE depending both on the players' category and the digital orientation.

Research limits: This paper is not exempt from limitations, especially related to the qualitative and exploratory nature of the study, and the results cannot be generalised.

Practical implications: The results offer useful suggestions to the different players studied (clicks-and-mortar, pure play enterprises, digital and traditional agencies) with the aim to improve the effectiveness of the CBE strategies.

Originality of the paper: Previous research on CBE mainly focused on the analysis of the consumer's point of view. Focusing on different types of enterprises and agencies, our study provides the first academic contribution to the understanding of the managerial point of view within an original conceptual framework that integrates multiple key issues faced in developing CBE strategies.

Key words: Consumer-brand engagement; traditional and digital agency; 'clicksand-mortar' and 'pure play' firm

\section{Introduzione}

L'evoluzione delle condizioni di mercato, competitive e tecnologiche, nonché il cambiamento paradigmatico che interessa la comunicazione di marketing, stanno determinando la crescente rilevanza e articolazione del concetto di engagement nell'ambito delle strategie d'impresa (Hollebeek et al., 2016a). In particolare, i marketing manager cercano di sperimentare strategie in grado di sviluppare relazioni di maggior valore con i clienti attuali e potenziali, che vadano ben oltre l'acquisto (Van Doorn et al., 2010). Ad esempio, il Marketing Science Institute considera il customer engagement: "customers' behavioral manifestation toward a brand or firm beyond purchase" (MSI, 2010, p. 4). L'obiettivo delle imprese diviene quello di costruire, attraverso l'esperienza nei diversi touch point, livelli più profondi di legame basati sull'engagement (Islam e Rahman, 2016; Venkatesan, 2017), in modo tale da iniziare a configurare un vero e proprio "orientamento strategico all'engagement” (Kumar e Pansari, 2016). Soprattutto a seguito dello sviluppo dei media digitali, anche i consulenti della comunicazione portano avanti un incisivo lavoro volto a creare consapevolezza, educando i loro clienti sul tema (Anderson, 2015), tanto che ormai tra i professionisti il termine "engagement" è diventato una "buzzword", un termine alla moda, che tuttavia presenta contorni ancora piuttosto incerti (Kozinets, 2014). Dal lato dell'accademia, gli studiosi hanno altresì aumentato gli sforzi per delineare sempre meglio non solo le dimensioni concettuali fondanti dell'engagement e le sue diverse "declinazioni" (es. customer/ consumer engagement, consumer-brand engagement, employee engagement, stakeholder engagement), ma anche i nessi che lo legano agli altri costrutti relazionali più tradizionali, come la soddisfazione, la fiducia e la loyalty (Pansari e Kumar, 2017), nel tentativo di fare luce sugli antecedenti e sulle 
variabili-risultato (Brodie et al., 2011; Hollebeek et al., 2014). Difatti, il "customer engagement" è stato indicato dal Marketing Science Institute tra le "research priorities" sia per i periodi 2006-2008, 2010-2012 che per il 2014-2016, oltre ad essere stato posto al centro di special issue da parte di primarie riviste internazionali, quali: Journal of the Academy of Marketing Science (2017), Journal of Service Research (2010, 2011) e Journal of Consumer Psychology (2009). Sebbene lo sviluppo di queste linee di ricerca sia ancora in fieri, vi è un diffuso accordo nel ritenere che il "customer" o "consumer" engagement rappresenti oggi "un imperativo strategico" (Brodie et al., 2011, p. 252), al fine di migliorare le performance economiche e competitive sia nel B-to- $\mathrm{C}$ che nel B-to- $\mathrm{B}$, contribuendo, ad esempio, a: raggiungere un vantaggio competitivo distintivo (Kumar e Pansari, 2016), accrescere le vendite (Neff, 2007), migliorare la quota di mercato e la profittabilità (Hoyer et al., 2010). Più nello specifico, oggi, il "consumer-brand engagement" (d'ora in avanti anche CBE) sembra giocare un ruolo centrale nelle strategie di branding (Gambetti e Graffigna, 2010). A fronte della rilevanza sotto il profilo manageriale di questo concetto, la letteratura accademica finora si è concentrata prevalentemente sullo studio della prospettiva del consumatore/cliente, al fine di comprendere quali possano essere le differenti dinamiche alla base dello sviluppo dell'engagement da parte di un individuo verso uno specifico oggetto, quale un brand, un'organizzazione o un particolare media. Tuttavia, come evidenziato anche da un recente contributo di Hollebeek et al. (2016a), una delle aree chiave per il futuro avanzamento degli studi in questo ambito riguarda le modalità di gestione del customer/consumer engagement in un contesto multimedia e multichannel, assumendo la prospettiva delle imprese. Di conseguenza, la finalità del presente lavoro è di contribuire a rispondere a questa esigenza conoscitiva e a colmare il gap della letteratura, analizzando le prospettive manageriali relative in particolare alle strategie di consumer-brand engagement all'interno di un originale framework concettuale integrato. Quest'ultimo è stato sviluppato in modo induttivo attraverso lo studio empirico del punto di vista di due tra le principali categorie di player, che sono oggi responsabili dello sviluppo di iniziative di marketing e comunicazione focalizzate sull'engagement, ossia gli inserzionisti (imprese "clicks-and-mortar" e "pure play") e le agenzie di comunicazione (tradizionali e digitali).

Il paper si articola come segue: nel paragrafo successivo, si proporrà una sintesi del background teorico sul CBE, da cui discendono le specifiche domande di ricerca alle quali intendiamo rispondere; verranno quindi presentati il metodo seguito e i principali risultati emersi. Infine, il lavoro si concluderà con la discussione dei risultati, la presentazione delle implicazioni manageriali e accademiche, la segnalazione dei limiti e l'identificazione delle future linee di ricerca.

\section{Background teorico e domande di ricerca}

Prima di essere posto al centro dell'attenzione da parte degli studiosi di marketing, il concetto di "engagement" è stato studiato in altre discipline
Maria Vernuccio Gabriele Qualizza Andrea Buratti Federica Ceccotti Strategie di consumer-brand engagement. Il punto di vista delle imprese e delle agenzie di comunicazione 
sinergie Vol. 36, N. 106, 2018

accademiche, come la psicologia, il comportamento organizzativo e l'information science (Hollebeek, 2011; Vivek et al., 2012). A partire dal 2005, il termine "customer/consumer engagement" ha iniziato ad essere utilizzato anche nella letteratura di marketing e si è imposto gradualmente in questo ambito come un filone di ricerca molto rilevante e promettente, con un intensificarsi degli studi negli anni più recenti. Come evidenziato anche da una prima analisi sistematica della letteratura condotta sul tema da Islam e Rahman nel 2016, gli studiosi di marketing hanno approfondito il tema dellengagement adottando diverse teorie e prospettive di analisi. Le principali teorie utilizzate come framework concettuali di base per esplorare il "customer" o "consumer" engagement sono state il marketing relazionale (Carter, 2008; Bowden, 2009; Verhoef et al., 2010; Vivek et al., 2014) e la "Service-dominant (S-D) logic" (Vargo e Lush, 2004, 2008, 2016). In entrambe le teorie, $i$ consumatori non sono considerati come riceventi passivi dei messaggi di marca, ma al contrario come soggetti proattivi che contribuiscono alle interazioni incentrate sul brand (Fournier, 1998; Vargo e Lush 2008). Da qui, la base teorica che ha portato a considerare la natura altamente interattiva del concetto di customer/consumer engagement (Hollebeeck et al., 2016b). Altre basi teoriche del customer/consumer engagement sono relative al gruppo delle "social behavior theories", come ad esempio la "social exchange theory" (Blau, 1964; Hollebeek, 2011), e a quello delle "personal behavior theories", come la "affordance theory" (es. Cabiddu et al., 2014). Quale che sia la principale prospettiva teorica adottata, un elemento di fondamentale accordo sottostante ai diversi contributi è che "Engagement results from experiencing a brand as related to some higher-order personal life goal or value" (Calder et al., 2016a, p. 580). Fino ad oggi, sono già rintracciabili in letteratura numerosi contributi volti alla definizione del "customer/consumer engagement" (Hollebeek et al., 2014). Una possibile suddivisione è tra gli studiosi che ritengono che questo concetto si debba considerare più alla stregua di un processo psicologico e quelli che, invece, pongono maggiormente l'enfasi sul comportamento. Tra i primi Bowden (2009), che lo ha definito come un processo psicologico alla base dello sviluppo della fedeltà della clientela. Ancora, Mollen e Wilson (2010) definiscono l'engagement come quell'impegno - sia cognitivo che affettivo - da parte del consumatore nell'attivare una relazione con il brand. Secondo Brodie et al. (2011), il customer engagement è "a psychological state that occurs by virtue of interactive customer experiences with a focal object (e.g., a brand) in service relationships" (p. 260). Invece, altri autori, come Van Doorn et al. (2010) hanno analizzato la dimensione comportamentale dellengagement verso un brand o un'impresa (es. scrittura di review, passaparola), dimensione che può tradursi o meno nell'acquisto. Su questa linea di pensiero anche Jaakkola e Alexander (2014), che hanno studiato il ruolo del "customer engagement behavior" nella co-creazione di valore. La dimensione comportamentale e quella sociale rappresentano la natura proattiva ed interattiva del customer engagement, che si traduce in un'intensa partecipazione al discorso della marca e in una condivisione "fattiva" dei suoi valori. Ad ogni modo, a prevalere è la visione del "customer/consumer engagement" come di un concetto multi-dimensionale, che abbraccia le dimensioni cognitive, affettive, comportamentali e sociali (Gambetti et al., 
2012). Adottando tale prospettiva, Hollebeek et al. (2014, p. 151) hanno definito l'engagement come: "consumer's positively valenced cognitive, emotional and behavioural brand-related activity during, or related to, specific consumer/brand interactions". Come evidenziato da diversi autori, tra cui Brodie et al. (2011), il customer engagement dovrebbe essere considerato in relazione al contesto specifico ("it occurs under a specific set of contextdependent conditions", p. 260) e all'oggetto verso il quale l'individuo sviluppa l'engagement ("focal object", p. 260). Riguardo al contesto, prevalgono gli studi svolti nell'ambito delle piattaforme online (es. siti web, social network, online brand community) e in quello dei servizi, anche se sono numerose le ricerche che abbracciano trasversalmente molteplici contesti (Islam e Rahman, 2016). Con riferimento all'"oggetto focale", in letteratura si rinvengono principalmente due differenti prospettive. Da un lato, viene approfondito l'engagement verso la pubblicità e i media (Calder et al., 2016b; Calder e Malthouse, 2005; Calder et al., 2009; Malthouse et al., 2007; Mersey et al., 2010). In altri studi, invece, viene analizzato il customer o consumer engagement verso il brand (Graffigna e Gambetti, 2015; Gambetti et al., 2012; Hollebeek, 2011; Hollebeck et al., 2014; Sprott et al., 2009). Il nostro studio si inserisce in questo secondo filone di ricerca, che finora ha accolto contributi empirici e teorici finalizzati prevalentemente a chiarire la concettualizzazione del CBE e a far luce sulle principali dimensioni - cognitive, emozionali, comportamentali, esperienziali e sociali (Gambetti et al., 2012) - nelle quali esso può essere declinato.

Eccezion fatta per i citati lavori esplorativi di Gambetti et al. (2012) e di Vivek et al. (2012), la maggior parte degli studi empirici è stata focalizzata sulla prospettiva del consumatore. Tuttavia, a fronte del crescente impegno profuso dalle imprese nella sperimentazione di strategie volte a creare CBE attraverso i diversi touch point, online come offline (Venkatesan, 2017), riteniamo che, a fianco della prospettiva del consumatore, sia importante avviare un sistematico approfondimento del punto di vista dei manager. A tal proposito, di recente Kumar e Pansari (2016) sottolineano l'utilità di indirizzare la futura ricerca su quella che definiscono "engagement orientation", intesa come: "the process of embedding engagement in the organization as a policy decision and ensuring that all strategies of the organization focus on engaging the customers and the employees, along with value maximization for all stakeholders" (p. 511). Il presupposto è il passaggio da una prospettiva diadica a una visione più ampia, orientata ad abbracciare, come suggerito da Brodie et al. (2016), le più ampie reti di relazioni in cui ha luogo l'incontro tra consumatore e brand. Si tratta di un fitto intreccio di interazioni tra una molteplicità di stakeholder e di attori: fornitori, partner in affari, collaboratori, canali distributivi, comunità di consumatori, singoli clienti, ecc. (Chandler e Lusch, 2015; Jaakkola e Alexander, 2014; Lemon e Verhoef, 2016; Tax et al., 2013). Si può parlare a questo proposito di "engagement ecosystems" (Breidbach et al., 2014; Brodie et al., 2016) o anche di eco-sistemi di esperienze, ciascuno dei quali può essere definito come: "the complex set of relationships among a company's employees, partners and customers that determines the quality of all customer interactions" (Bodine et al., 2013, p. 7). Le interazioni tra i
Maria Vernuccio Gabriele Qualizza Andrea Buratti Federica Ceccotti Strategie di consumer-brand engagement. Il punto di engagement. Il punto di
vista delle imprese e delle agenzie di comunicazione 
sinergie Vol. 36, N. 106, 2018

componenti di questi network sono non lineari e multi-direzionali: questo significa che possono essere intraprese da qualunque attore e che ogni azione di un elemento sullaltro ha come conseguenza una reazione da parte non solo del destinatario, ma anche dell'intero ecosistema (Maslowska et al., 2016).

Inoltre, pur riconoscendo la natura "context-specific" dellengagement, riteniamo che sia estremamente utile iniziare a considerare questo concetto anche in modo più ampio, sotto il duplice profilo teorico e delle implicazioni manageriali. Di conseguenza, la nostra ricerca si propone di adottare una chiave di lettura differente del CBE, ossia quella più ampia dei principali attori del communication network (Ceccotti, 2018; Vernuccio e Ceccotti, 2015, 2017): da una parte, le imprese inserzioniste (d'ora in avanti, anche "imprese"), "clicks-and-mortar" e "pure play"1, e dallaltra, le agenzie di comunicazione, tradizionali e digitali. La finalità è quella di individuare ed analizzare i principali temi manageriali con riferimento alle strategie di CBE all'interno di un originale framework concettuale integrato. Quest'ultimo verrà sviluppato induttivamente attraverso una ricerca esplorativa di tipo qualitativo, che consentirà di far emergere le similarità nella molteplicità dei punti di vista $\mathrm{e}$, al contempo, di riconoscere alcune specificità di visione delle diverse categorie di player, in virtù dei loro differenti obiettivi di business, nonché della diversità delle informazioni detenute e del livello di esperienza nel campo specifico (Grant et al., 2012). Appare rilevante non solo il confronto inter-categoria tra imprese e agenzie, impegnate a collaborare nel ruolo di investitori e consulenti nello sviluppo di strategie di CBE, ma anche quello intra-categoria, ovvero tra imprese clicks-and-mortar e pure play, da una parte, e tra agenzie tradizionali e digitali, dall'altra. Potrebbe essere, infatti, interessante comprendere se e come il diverso orientamento al digital, ad esempio in termini di livello di concentrazione del business nel mercato digitale (Chaffey et al., 2009) e di possesso di competenze digitali (Vernuccio e Ceccotti, 2015), possa influenzare una diversa lettura manageriale del CBE.

La finalità di ricerca sopra richiamata è stata declinata nelle seguenti domande di ricerca:

RQ1 - Quali sono i significati del CBE secondo le agenzie di comunicazione (tradizionali e digitali) e gli inserzionisti (imprese clicksand-mortar e pure play)?

$R Q 2$ - Quali sono gli obiettivi manageriali che le agenzie (tradizionali e digitali) e gli inserzionisti (imprese clicks-and-mortar e pure play) intendono conseguire attraverso lo sviluppo di strategie di CBE?

RQ3 - Quali sono i sistemi manageriali (attività e strumenti) considerati come più importanti per lo sviluppo del CBE secondo le agenzie (tradizionali e digitali) e gli inserzionisti (imprese clicks-and-mortar e pure play)?

Con il termine "clicks-and-mortar" qualifichiamo quelle imprese inserzioniste che nascono per operare offline e, in seguito, avviano un business online, combinando in tal modo una presenza sul mercato di tipo ibrido, ossia online e offline (es. Ikea). Viceversa, le imprese "pure play" nascono per operare prevalentemente online, talvolta attivando dei punti di contatto anche offline (es. Amazon) (Afuah e Tucci, 2001; Chaffey et al., 2009). 
$R Q 4$ - Quali sono le principali sfide manageriali legate allo sviluppo del CBE secondo le agenzie (tradizionali e digitali) e gli inserzionisti (imprese clicks-and-mortar e pure play)?

\section{Metodologia}

La natura delle domande di ricerca, unitamente all'assenza di studi precedenti, hanno reso opportuna l'adozione di un approccio esplorativo ed induttivo (Armstrong, 1979) di tipo qualitativo. Lambito di elezione per l'utilizzo dei metodi qualitativi è quello solitamente definito come "contesto della scoperta", data la loro capacità di far emergere il nuovo, portando alla luce significati e nessi inaspettati: lobiettivo non è controllare deduttivamente ipotesi formulate in precedenza, bensì generare induttivamente chiavi interpretative applicabili a fenomeni ancora poco indagati (Delli Zotti, 2004; Reichardt e Cook, 1979).

Nella fase pilota, sono stati intervistati cinque soggetti con una significativa esperienza a livello internazionale nei settori della comunicazione di marketing e del branding, al fine di migliorare l'efficacia della traccia d'intervista, che è stata articolata sulla base delle quattro domande di ricerca sopra illustrate. Successivamente, sono state condotte 39 interviste in profondità (Creswell, 1998) a marketing manager ( $\mathrm{n}=22)$ e consulenti di comunicazione ( $\mathrm{n}=17)$ appartenenti a: 1$)$ agenzie tradizionali $(\mathrm{n}=10)$, agenzie digitali $(\mathrm{n}=12)$, imprese clicks-and-mortar $(\mathrm{n}=10)$ e pure play $(\mathrm{n}=7)$, come risulta dalla tabella 1 . Le organizzazioni in target sono state definite selezionando imprese/agenzie leader nei differenti settori di riferimento (tabella 1), avendo cura di diversificare il set d'indagine, al fine di garantire la varietà dei punti di vista. I manager e i professionisti da includere nel set d'indagine sono stati individuati seguendo il "key informant approach" (Robson e Foster, 1989). Sono stati contattati e intervistati quasi esclusivamente soggetti con ruolo aziendale elevato. Ad esempio: Marketing manager, Digital Communication manager, Online marketing \& PR manager, per la categoria imprese inserzioniste; CEO, Web marketing manager, General manager, Senior vice President, per la categoria agenzie. Inoltre, è stato considerato come criterio di selezione l'esperienza maturata nel campo disciplinare della nostra indagine. Gli intervistati, 31 uomini e 8 donne, hanno unesperienza variabile da 10 a 30 anni e lavorano per organizzazioni operanti a livello internazionale.

La dimensione del set dosservazione è stata definita mediante il criterio della "saturazione teorica" (Cardano, 2003; Corbin e Strauss, 2008; Glaser e Strauss, 1967): la raccolta dei dati è proseguita fino al momento in cui si è raggiunto un livello di "redundancy", per cui l'apporto di ulteriori interviste sarebbe risultato nullo o estremamente modesto rispetto agli obiettivi conoscitivi del lavoro.
Maria Vernuccio Gabriele Qualizza Andrea Buratti Federica Ceccotti Strategie di consumer-brand engagement. Il punto di vista delle imprese e delle agenzie di comunicazione 


\section{sinergie}

Vol. 36, N. 106, 2018
Tab. 1: Il set d'indagine

\begin{tabular}{|c|c|c|c|}
\hline $\begin{array}{l}\text { Macro-categorie } \\
\text { di operatori }\end{array}$ & \begin{tabular}{|c|}
$\begin{array}{c}\text { Tipologia di Impresa/ } \\
\text { Agenzia }\end{array}$ \\
\end{tabular} & $\begin{array}{l}\text { Settore (Numero di } \\
\text { partecipanti) }\end{array}$ & Ruolo dei rispondenti \\
\hline \multirow{15}{*}{$\begin{array}{l}\text { Imprese } \\
\text { inserzioniste } \\
(\mathrm{n}=17)\end{array}$} & \multirow{9}{*}{$\begin{array}{l}\text { Imprese } \\
\text { clicks-and-mortar } \\
(\mathrm{n}=10)\end{array}$} & Luxury $(\mathrm{n}=1)$ & Brand Manager \\
\hline & & Automotive $(\mathrm{n}=1)$ & Marketing Manager \\
\hline & & Alimentare $(\mathrm{n}=1)$ & Digital Marketing Manager \\
\hline & & Energia $(n=1)$ & Web media Relations Manager \\
\hline & & Non profit $(n=1)$ & Digital Strategic Planner \\
\hline & & Intrattenimento $(\mathrm{n}=2)$ & $\begin{array}{l}\text { Responsabile Comunicazione } \\
\text { e Promozione, Responsabile } \\
\text { Online Marketing, Social media } \\
\text { \& CRM }\end{array}$ \\
\hline & & Servizi finanziari $(\mathrm{n}=1)$ & Marketing Manager \\
\hline & & Farmaceutico $(\mathrm{n}=1)$ & $\begin{array}{l}\text { Digital Communication } \\
\text { Manager }\end{array}$ \\
\hline & & $\begin{array}{l}\text { Telecomunicazioni } \\
(\mathrm{n}=1)\end{array}$ & Business Analyst \\
\hline & \multirow{6}{*}{$\begin{array}{l}\text { Imprese pure play } \\
(\mathrm{n}=7)\end{array}$} & Turismo $(\mathrm{n}=1)$ & $\begin{array}{l}\text { Digital PR \& Media Relations } \\
\text { Manager }\end{array}$ \\
\hline & & Social Network $(\mathrm{n}=1)$ & $\begin{array}{l}\text { Head of Marketing Southern } \\
\text { Europe }\end{array}$ \\
\hline & & \begin{tabular}{|l|}
$\begin{array}{l}\text { Comparatore di prezzi } \\
(\mathrm{n}=1)\end{array}$ \\
\end{tabular} & \begin{tabular}{|l|} 
Online marketing \& PR \\
Manager
\end{tabular} \\
\hline & & Luxury $(\mathrm{n}=2)$ & $\begin{array}{l}\text { Global Head of Performance } \\
\text { Marketing, } \\
\text { Customer engagement \& } \\
\text { Innovation Specialist } \\
\end{array}$ \\
\hline & & \begin{tabular}{|l|} 
Abbigliamento e \\
accessori $(\mathrm{n}=1)$ \\
\end{tabular} & $\begin{array}{l}\text { Online Marketing Manager \& } \\
\text { Co-founder }\end{array}$ \\
\hline & & \begin{tabular}{|l}
$\begin{array}{l}\text { Wearable Technologies } \\
(\mathrm{n}=1)\end{array}$ \\
\end{tabular} & $\mathrm{CEO}$ \\
\hline \multirow{6}{*}{$\begin{array}{l}\text { Agenzie di } \\
\text { comunicazione } \\
(\mathrm{n}=22)\end{array}$} & \multirow{3}{*}{$\begin{array}{l}\text { Agenzie } \\
\text { digitali } \\
(\mathrm{n}=12)\end{array}$} & $\begin{array}{l}\text { Digital Communication } \\
(\mathrm{n}=7)\end{array}$ & $\begin{array}{l}\text { Marketing Director, Account } \\
\text { Director, Social media Manager, } \\
\text { Co-Founder \& CEO, Web } \\
\text { Marketing Strategist, Office } \\
\text { Director, General Manager } \\
\end{array}$ \\
\hline & & \begin{tabular}{|l|}
$\begin{array}{l}\text { Digital Monitoring } \\
(\mathrm{n}=1)\end{array}$ \\
\end{tabular} & $\mathrm{CEO}$ \\
\hline & & Digital Marketing $(\mathrm{n}=4)$ & $\begin{array}{l}\text { Consultant, Web Marketing } \\
\text { Manager, Web Marketing } \\
\text { Specialist, Digital PR \& } \\
\text { Community Manager } \\
\end{array}$ \\
\hline & \multirow{3}{*}{$\begin{array}{l}\text { Agenzie } \\
\text { tradizionali } \\
(\mathrm{n}=10)\end{array}$} & Centro media $(\mathrm{n}=1)$ & Sales Account Manager \\
\hline & & Pubblicità $(\mathrm{n}=6)$ & $\begin{array}{l}\text { CEO }(n=2), \text { Strategic Planner, } \\
\text { Community Manager, Account } \\
\text { Manager, Co-Founder and } \\
\text { Executive Vice President } \\
\end{array}$ \\
\hline & & $\begin{array}{l}\text { Pubbliche relazioni } \\
(\mathrm{n}=3)\end{array}$ & $\begin{array}{l}\text { CEO, Senior Vice President, } \\
\text { Senior Consultant }\end{array}$ \\
\hline
\end{tabular}

Fonte: nostra elaborazione

Le interviste hanno raggiunto una durata media di 45 minuti, sono state audio-registrate e quindi fedelmente trascritte, costruendo un data set di 256 pagine di trascrizioni (A4 in formato Word, interlinea doppia). L'individuazione dei concetti chiave all'interno del data set è stata realizzata attraverso l'applicazione della thematic content analysis 
(Boyatzis, 1998; Braun e Clarke, 2006; Langdridge, 2004). A differenza dellanalisi quantitativa dei contenuti, che tende a focalizzare l'attenzione a livello micro, isolando dal contesto semplici frasi o singole parole, con lobiettivo di ridurre ampie quantità di testo a dati numerici suscettibili di analisi statistica (Krippendorf, 2004), la thematic analysis richiede che il ricercatore non perda mai di vista il senso della conversazione nel suo insieme (Breidbach et al., 2014; Thomsen et al., 1998): per questo, prende in considerazione significative porzioni di testo, capaci di esprimere un concetto articolato (Braun e Clarke, 2006). Lanalisi qualitativa è stata supportata dal software Nvivo8 ${ }^{\circ}$, una soluzione che ha consentito di ottenere un output più rigoroso, anche a seguito di un processo di codifica di tipo double-blind (Bazeley, 2007). In accordo con King e Horrocks (2010), sono stati impiegati codificatori interni al team di ricerca, poiché questi hanno una conoscenza contestuale dei dati, che favorisce lo sviluppo di una più ampia e approfondita discussione per la definizione e larticolazione dei codici (temi). Il testo è stato suddiviso in segmenti di contenuto, che sono stati associati a temi, definiti induttivamente (a posteriori), attraverso unanalisi qualitativa del testo condotta separatamente da due degli autori. Nell'ambito delle più ampie aree tematiche definite dalle domande di ricerca, sono stati in tal modo interpretati i temi manageriali più rilevanti (King e Horrocks, 2010), che qualificano il pensiero dei rispondenti con riferimento ai significati, agli obiettivi, ai sistemi di gestione e alle sfide del $\mathrm{CBE}$. Conclusa questa fase, i risultati dei due processi di analisi tematica sono stati confrontati, discussi e armonizzati dai codificatori. L'affidabilità dei risultati è stata perseguita ricorrendo al cosiddetto metodo del "code-confirming": la codifica tematica del testo è stata verificata da due codificatori indipendenti, esperti di marketing e specificamente formati sotto il profilo metodologico, i quali hanno confermato o meno le associazioni individuate tra testi e temi. La percentuale di accordo tra $\mathrm{i}$ codificatori è risultata pari all' $88 \%$, superiore al livello minimo previsto nelle ricerche esplorative (Powell, 2007). Infine, per quanto riguarda la "credibilità" dei risultati dell'analisi tematica, questi sono stati sottoposti all'attenzione di tre soggetti del set d'indagine, i quali li hanno condivisi e "validati" (Lincoln e Guba, 1985).

\section{Analisi dei risultati}

In linea con le domande di ricerca, i risultati dell'analisi qualitativa del contenuto si articolano in quattro aree tematiche (Fig. 1): 1) i significati del CBE $(\$ 4.1), 2)$ gli obiettivi manageriali delle strategie di CBE $(\$ 4.2)$, $3)$ i sistemi manageriali del CBE ( $\$ 4.3)$ e, infine, 4 ) le sfide manageriali del CBE (\$ 4.4).

La Fig. 1 rappresenta il framework concettuale integrato emerso della nostra analisi, che evidenzia i concetti principali (temi) e le relazioni esistenti tra questi, per cui si è registrato un significativo livello di convergenza nelle risposte degli intervistati. Nellambito di ciascuno dei temi, sono stati individuati dei sotto-temi, in relazione ai quali sono emerse alcune specificità di prospettiva. Nella presentazione dei risultati
Maria Vernuccio

Gabriele Qualizz

Andrea Buratti

Federica Ceccotti

Strategie di consumer-brand

engagement. Il punto di

vista delle imprese e delle agenzie di comunicazione 
sinergie italian journal of management Vol. 36, N. 106, 2018

di seguito, dunque, saranno evidenziate le differenze esistenti tra $\mathrm{i}$ rispondenti appartenenti alle diverse categorie (agenzia versus impresa) e, all'interno della stessa categoria, eventuali specificità di visione derivanti da un maggiore o minore orientamento al digital (ad esempio, nellambito della categoria imprese, tra clicks-and-mortar e pure play).

Fig. 1: Le strategie di CBE nella prospettiva manageriale: un framework concettuale integrato

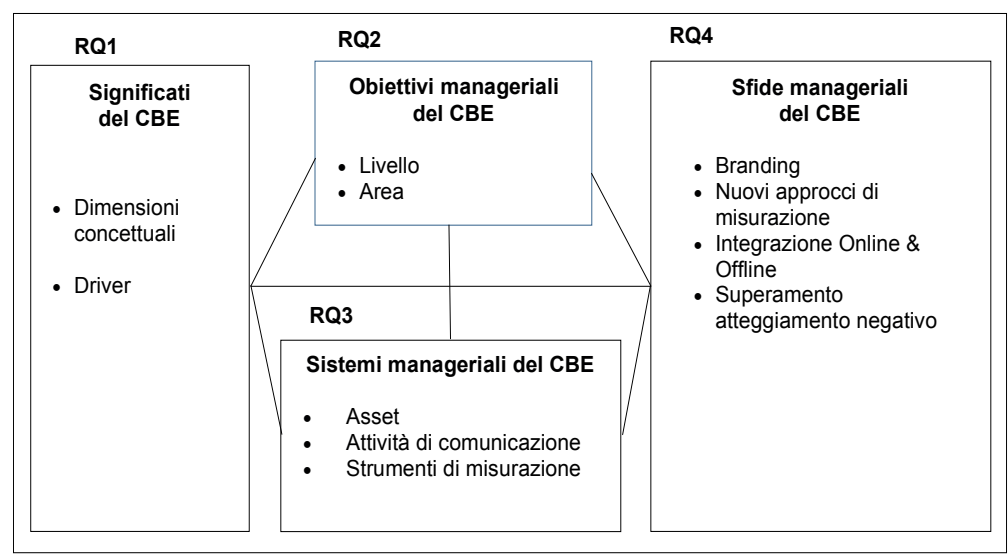

Fonte: nostra elaborazione

\subsection{I significati del CBE (RQ1)}

Con specifico riferimento ai significati del CBE, dalle risposte degli intervistati sono emersi due temi - le dimensioni concettuali e i driver del consumer-brand engagement - a loro volta articolati in differenti sotto-temi (Fig. 2).

\section{Dimensioni concettuali del CBE}

Tuttigli intervistati, indipendentemente dalla categoria di appartenenza, concordano nel riconoscere che alla base del CBE vi sia il concetto di relazione interattiva e dinamica. Tuttavia, tale relazione dinamica può essere interpretata come la combinazione variabile di differenti dimensioni concettuali, ossia (Fig. 2):

- dimensione interattiva diadica, riferita all'interazione tra consumatore e brand (one-to-one);

- dimensione interattiva sociale, legata alle interazioni tra consumatori e tra questi e il brand in una logica many-to-many (consumers-toconsumers-to-brand);

- dimensione partecipativa, inerente al contributo in termini di contenuti che l'utente fornisce partecipando attivamente ai "discorsi" legati al brand;

- dimensione di identificazione del consumatore nel brand, riguardante la percezione di "overlapping" tra l'identità dell'individuo e quella della marca; 
- dimensione emozionale, riferita al legame "affettivo" che l'individuo prova verso il brand;

- dimensione cognitiva, legata alla conoscenza, all'interesse e allattenzione nei confronti del brand.

Fig. 2: I significati del CBE: temi e sotto-temi
Maria Vernuccio Gabriele Qualizza Andrea Buratti Federica Ceccotti

Strategie di consumer-brand

engagement. Il punto di vista delle imprese e dell agenzie di comunicazione

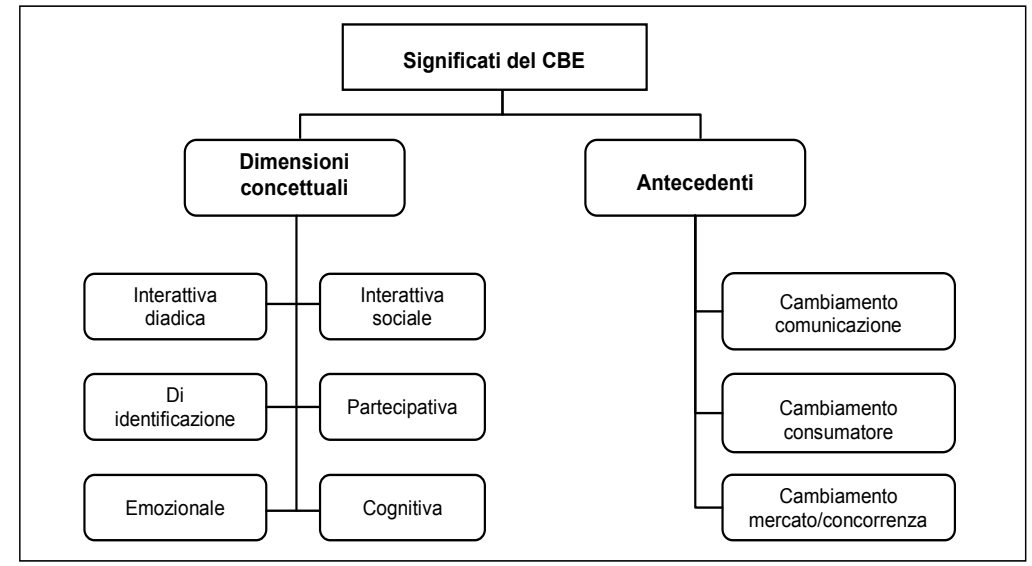

Fonte: nostra elaborazione

Con riferimento a tali dimensioni concettuali (sotto-temi), possono essere messe in luce alcune differenze nei punti di vista tra le categorie oggetto d'analisi. A tal proposito, possiamo sottolineare come le agenzie dimostrano di avere la visione più articolata dei significati del CBE, richiamando tutte le differenti dimensioni concettuali dello stesso. Meno consapevoli della complessità sottostante al CBE sono, invece, le imprese, che si focalizzano soprattutto sulla componente interattiva diadica dell'engagement, dando prevalente enfasi al coinvolgimento diretto brandconsumatore.

"L'engagement prevede l'interazione diretta con i consumatori, realizzata prevalentemente attraverso i social media e più raramente attraverso altri punti di contatto. Sui social non solo sono possibili forme di interazione superficiali, come un semplice like, ma anche conversazioni vere e proprie tra marca e consumatore" (Responsabile Online Marketing, Social media \& CRM, Impresa clicks-and-mortar).

Interessante è l'analisi delle specificità all'interno delle differenti categorie. Infatti, nelle risposte degli intervistati appartenenti alle agenzie emergono alcune distinzioni: se quelle tradizionali colgono, in aggiunta alla dimensione interattiva diadica, soprattutto gli aspetti emozionali e di identificazione con il brand, le agenzie digitali sono quelle che hanno la visione più completa della complessità relazionale connessa al CBE, riconoscendo, oltre alla dimensione interattiva diadica, anche quella sociale, partecipativa, emozionale e cognitiva.

(CBE significa) "Rendere rilevante la marca per il consumatore e rendere protagonista il consumatore e coinvolgerlo nella filosofia di marca in modo che diventino un unicum. Ognuno entra nella filosofia di vita dell'altro: le 
sinergie Vol. 36, N. 106, 2018

mie marche fanno parte della mia vita e della mia esperienza e io allo stesso tempo faccio parte del mondo della marca, perché posso condividere dei valori con lei" (Account manager, Agenzia tradizionale).

Anche nellambito della categoria impresa, si riscontrano delle specificità: pur riconoscendo tutte le dimensioni del fenomeno - eccezion fatta per quella cognitiva - le imprese pure play sembrano concentrarsi sull'interattività diadica, mentre quelle clicks-and-mortar abbracciano anche la dimensione emozionale e quella sociale.

"Per noi il 'consumer-brand engagement' è la creazione di un legame, un dialogo personale con il cliente, che ha un impatto su loyalty e fiducia verso il brand. Il cliente viene approcciato in maniera individuale, in modo da farlo sentire parte dell'universo del brand (Customer engagement \& Innovation Specialist, Impresa pure play).

\section{Driver del CBE}

Nelle risposte degli intervistati, le principali cause di sviluppo delle strategie di CBE sono riconducibili a tre sotto-temi principali (Fig. 2):

- il cambiamento della comunicazione, che dagli intervistati viene legato alla crisi dei modelli tradizionali di comunicazione (basati su una logica push), alla diffusione dei media digitali e dei social media, alla moltiplicazione dei touch point e, infine, alla nascita di nuovi paradigmi comunicativi di gestione del brand;

- il cambiamento del consumatore e del processo di acquisto, nel segno di crescenti livelli di consumer empowerement, iperconnessione e comportamento multicanale;

- il cambiamento del mercato e della concorrenza, questi ultimi sempre più dinamici e difficili da interpretare.

Interessanti sono le differenze emerse inter-categoria. Infatti, le agenzie attribuiscono la crescita di importanza del CBE principalmente al cambiamento della comunicazione e vivono tale cambiamento come un salto paradigmatico, legato alla crisi dei modelli tradizionali, alla diffusione dei media digitali, alla moltiplicazione dei touch point e ai nuovi paradigmi di gestione della marca. Per le imprese, invece, le motivazioni della crescente rilevanza del CBE sono più varie: non sono, infatti, esclusivamente legate al cambiamento della comunicazione - che, peraltro, riconducono essenzialmente allo sviluppo dei media digitali - ma anche a quello del consumatore e del mercato/concorrenza.

A livello intra-categoria, si riscontrano ulteriori differenze di prospettiva. In particolare, oltre al cambiamento nella comunicazione, le agenzie tradizionali e le imprese clicks-and-mortar ritengono importante il cambiamento del mercato e della concorrenza, mentre le agenzie digitali e le imprese pure play attribuiscono molta importanza al cambiamento del consumatore. Infine, una specificità delle imprese clicks-and-mortar è che leggono la rilevanza strategica assunta dallengagement più nel segno della continuità che non nell'ottica del cambiamento paradigmatico:

"Lengagement cè sempre stato... anche la conferenza stampa e la fiera possono essere engaging! Ma sono cambiati i mezzi, perché con lo sviluppo del digital si possono ottenere unesposizione e una reach molto più ampie rispetto al passato" (Marketing manager, Impresa clicks-and-mortar). 
Con riguardo agli obiettivi manageriali del CBE, le risposte hanno fatto emergere i temi del livello (o scope) e dell'area dell'obiettivo, declinati poi in engagement. Il punto di vista delle imprese e delle differenti sotto-temi (Fig. 3).

\section{Livello dellobiettivo (o scope)}

Per quel che concerne il tema dello "scope" degli obiettivi delle strategie di CBE, possono essere individuati tre livelli (sotto-temi) (Fig. 3):

- strategico;

- tattico;

- strategico e tattico, in modo integrato.

Fig. 3: Gli obiettivi manageriali delle strategie di CBE: temi e sotto-temi

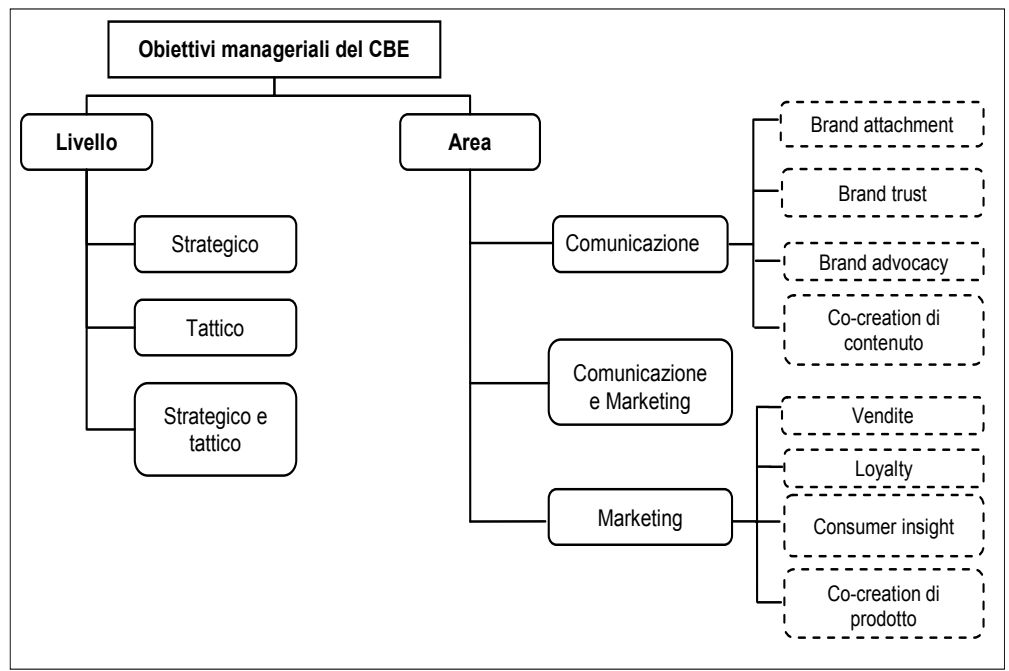

Fonte: nostra elaborazione

Nelle risposte delle diverse categorie emerge un sostanziale allineamento: sia per le agenzie che per le imprese il livello dell'engagement è soprattutto strategico, sebbene per le seconde questo possa caricarsi anche di una valenza esclusivamente tattica (come accade, ad esempio, nel caso del real-time marketing).

All'interno della categoria agenzie, si riscontrano alcune differenze: quelle digitali vedono il CBE come un obiettivo esclusivamente strategico nei progetti di comunicazione online, mentre per le agenzie tradizionali può anche trattarsi di un mix di strategia e tattica e si colloca al centro dell'intero ecosistema di comunicazione, che integra media online e offline.

"Dobbiamo lavorare su due piani, uno tattico - si pensi ai recenti casi di instant marketing su Twitter e Facebook - che sempre più necessiterà di un grande rapporto di fiducia tra agenzie e aziende, ed un piano strategico di lungo periodo, con obiettivi precisi (Co-Founder and Executive Vice President, Agenzia tradizionale). 
sinergie Vol. 36, N. 106, 2018

Anche le imprese clicks-and-mortar ritengono che, pure nel caso in cui lobiettivo del CBE sia di tipo tattico, questo debba essere sempre collegato al livello strategico. Lopinione è differente, infine, per le imprese pure play, per le quali il CBE può avere anche solo valenza tattica.

\section{Area dell'obiettivo}

Considerando più nel dettaglio il tema dellarea manageriale a cui afferiscono gli obiettivi delle strategie di CBE, sono state individuate tre aree (sotto-temi). Allinterno di queste, sono emersi numerosi obiettivi specifici associati al CBE, come indicato di seguito (Fig. 3):

- area degli obiettivi di comunicazione, a cui si possono ricondurre gli specifici obiettivi di brand attachment (da intendersi come forza del legame tra consumatore e brand), brand trust, brand advocacy e, infine, di co-creazione di contenuti;

- area degli obiettivi di marketing, più collegati alle vendite (nel breve e nel lungo termine), alla conoscenza del consumatore e alla co-creazione di prodotto. Rientrano in questa categoria le vendite, la brand loyalty, l'acquisizione di consumer insight e, infine, la co-creation di prodotto;

- aree di comunicazione e di marketing considerate in modo integrato.

Come per lo "scope" degli obiettivi, anche nell'ambito tematico dell'area manageriale, sembra prevalere a livello generale la convergenza di vedute tra gli attori: sia per le imprese che per le agenzie, vi è una maggioranza di rispondenti che considera l'obiettivo dellengagement rientrante al contempo nell'area marketing/vendite e in quella della comunicazione. $\mathrm{E}$ interessante, inoltre, andare più in profondità con l'analisi e considerare le similarità e le differenze di prospettiva che emergono tra i player con riferimento agli obiettivi di dettaglio associati alle strategie di CBE, da una parte, nell'area comunicazione e, dall'altra, in quella di marketing (si veda la Fig. 3).

Riguardo allarea marketing, emerge un importante tratto comune inter-categoria, ovvero la priorità della brand loyalty che, nel caso delle imprese, viene talvolta abbinata anche allobiettivo delle vendite nel breve periodo.

"Coinvolgendo il consumatore, si cerca di diminuire i tradimenti del consumatore con altre marche e di fidelizzarlo in maniera forte e duratura" (Account Manager, Agenzia tradizionale).

"E qualcosa che va oltre la semplice conclusione della vendita. Lengagement si crea quando il cliente percepisce un senso di appartenenza all'universo del brand e si lega alla marca nel tempo (Customer engagement \& Innovation Specialist, Impresa pure play).

Le agenzie, tuttavia, mostrano tendenzialmente una maggiore consapevolezza di tutti i possibili obiettivi di marketing. Emergono, inoltre, alcune differenze tra agenzie digitali e tradizionali: queste ultime si concentrano molto sulla brand loyalty e sulla co-creation di prodotto, mentre quelle digitali hanno una visione più articolata e dimostrano un orientamento consumer-centric ( $\$ 4.1$ ), considerando la raccolta di insight sul consumatore tra gli obiettivi più rilevanti. 
"L’engagement dà la possibilità di conoscere il proprio cliente, poiché questi, se coinvolto, è più attivo e quindi si espone, parla di sé... e laspetto più importante è che questa conoscenza della reale percezione delle persone non proviene da ricerche di mercato o da focus group, ma si costruisce attraverso la voce diretta delle persone (Account Director, Agenzia digitale).

Maria Vernuccio Gabriele Qualizza Andrea Buratti Federica Ceccotti Strategie di consumer-brand engagement. Il punto di vista delle imprese e delle agenzie di comunicazione

Riguardo alle imprese, emerge la focalizzazione superiore delle aziende pure play sull'insight del consumatore, mentre per le imprese clicks-andmortar sembrano più importanti altri obiettivi di marketing, quali la vendita nel breve, l'insight e, in misura molto inferiore, la co-creation di prodotto.

Sul versante degli obiettivi di comunicazione, si possono sottolineare trasversalmente una certa attenzione al brand attachment e una modesta attenzione al tema della co-creation di contenuti, soprattutto per quel che riguarda le imprese. Le differenze inter-categoria risultano altrettanto evidenti. Infatti, tra gli obiettivi di comunicazione, le imprese sembrano molto concentrate sullobiettivo del brand trust (soprattutto le aziende pure play), tendendo a sottovalutare tutte le ulteriori opzioni; viceversa le agenzie, ancora una volta, mostrano una maggiore consapevolezza, considerando in modo più equilibrato i diversi obiettivi.

"Con il brand engagement si può costruire la fiducia, il brand attachment, l'advocacy... Il consumatore può anche contribuire a piattaforme per lo sviluppo di un nuovo prodotto e ad attività di assistenza tecnica dando un supporto agli altri consumatori (Social media Manager, Agenzia digitale).

Anche a livello intra-categoria emergono alcune specificità. Sul fronte delle agenzie, gli obiettivi di comunicazione ritenuti relativamente più importanti sono il brand attachment e l'advocacy. Tuttavia, mentre per le agenzie digitali è più rilevante l'advocacy, espressione di una risposta comportamentale del consumatore, per quelle tradizionali risulta più importante il brand attachment e, dunque, la risposta di tipo valutativorelazionale.

In linea generale, infine, l'obiettivo della co-creation, sebbene non venga considerato come prioritario, sembra interessare più le agenzie delle imprese. Inoltre, le agenzie tradizionali pensano più alla co-creazione di prodotto (es. co-design packaging, proposta di nuove versioni); viceversa, le agenzie digitali danno maggiore importanza a quella comunicativa (es. partecipazione a contest creativi su slogan, video-contest).

\subsection{I sistemi manageriali del CBE (RQ3)}

Relativamente ai sistemi di gestione del CBE, dall'analisi del contenuto sono stati individuati tre temi, a loro volta articolati in differenti sotto-temi (Fig. 4):

- gli asset (o presupposti sui quali far leva per lo sviluppo di CBE);

- le attività/strumenti di comunicazione;

- gli strumenti di misurazione. 
Sinergie

Vol. 36, N. 106, 2018
Fig. 4: I sistemi manageriali del CBE: temi e sotto-temi

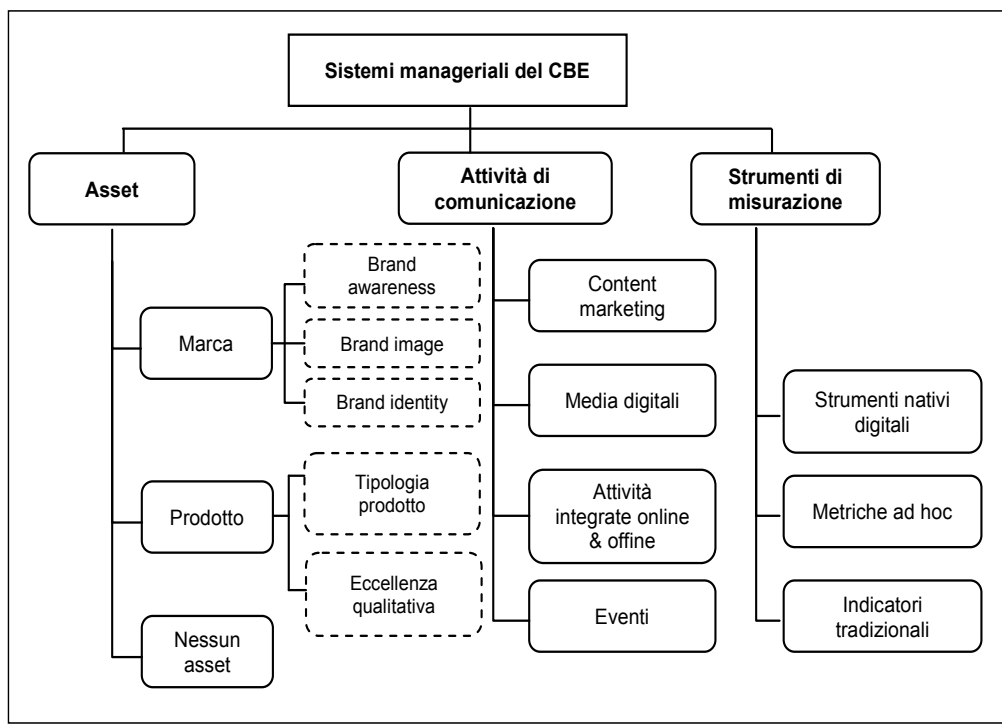

Fonte: nostra elaborazione

Asset

Il tema degli asset a partire dai quali è possibile sviluppare strategie di CBE di successo si articola nei seguenti sotto-temi:

- marca, declinata nei concetti di brand awareness, brand image e brand identity;

- prodotto, dal punto di vista della tipologia/categoria di prodotto e dell'eccellenza qualitativa;

- nessun asset in particolare come presupposto imprescindibile per sviluppare il CBE.

Tutte le categorie di imprese e agenzie si trovano d'accordo nel ritenere che sia la marca il principale asset sul quale far leva per sviluppare il CBE. Tuttavia, se da una parte le imprese considerano quale asset chiave l'immagine di marca, le agenzie, soprattutto digital, danno molta rilevanza anche alla notorietà. L'identità di marca, seppure considerata trasversalmente da tutti i rispondenti come un presupposto base, viene valutata come meno rilevante dell'immagine. Il prodotto, inoltre, riveste un'importanza relativa maggiore per le imprese, sia clicks-and-mortar che pure play, le quali pensano sia prioritario garantire l'eccellenza. Infine, secondo molti rispondenti di entrambe le categorie, non è necessario che vi sia un asset partenza, poiché il $\mathrm{CBE}$ si può sviluppare comunque, per qualsiasi brand e qualsiasi prodotto, attraverso strategie di marketing e comunicazione.

"Secondo me, il discorso dellengagement riguarda potenzialmente qualsiasi marca, di qualsiasi settore: tu puoi essere engaging con i frullatori, con l'elettronica, con il caffè solubile, eccetera. É chiaro che se sei la Ferrari o se hai un prodotto sexy, hai vita più facile, ma non hai l'esclusiva dell'engagement!' (CEO, Agenzia digital). 
Gabriele Qualizza

Lanalisi del contenuto ha portato a identificare quattro attivita/ Andrea Buratti strumenti di comunicazione considerati chiave nelle strategie di CBE (Fig. 4):

- il content marketing;

Strategie di consumer-brand

engagement. Il punto di

vista delle imprese e delle

- i media digitali;

- le attività di comunicazione integrata online-offline;

- gli eventi (offline).

Il principale dato comune inter-categoria è che i media digitali sono considerati gli strumenti più efficaci per il CBE. Ma mentre le agenzie si concentrano quasi esclusivamente sui social media, le imprese hanno una visione più ampia e considerano anche altri digital media, come il mobile e il sito web. Notevole convergenza vi è anche sull'importanza riconosciuta alle attività di comunicazione integrata tra online e offline. Tuttavia, va sottolineato come siano soprattutto le imprese clicks-and-mortar e le agenzie tradizionali a dare la priorità all'integrazione tra mondo fisico e virtuale, essendo minore la sensibilità di agenzie digitali e aziende pure play verso questa criticità.

“Cè bisogno di portare l'esperienza del consumatore dal contesto online a quello offline, in negozio... dove è possibile vedere e toccare il prodotto dal vivo. Con la strategia di engagement si cerca di portare a casa il cliente, combinando dimensione digitale e dimensione tradizionale" (Brand Manager, Impresa clicks-and-mortar).

Un'interessante differenza inter-categoria, riguarda il content marketing e gli eventi offline: le attività di content marketing sono percepite come centrali per il CBE esclusivamente dalle agenzie, mentre gli eventi vengono valutati rilevanti solo dalle imprese, soprattutto clicks-and-mortar.

\section{Strumenti di misurazione}

Il tema degli strumenti di misurazione del CBE è stato articolato a sua volta nei seguenti sotto-temi:

- strumenti nativi digitali, ossia i tool (con delle metriche standard) messi a disposizione dei publisher dai principali player digitali, come Google Analytics e Facebook Insight;

- metriche ad hoc, ovvero sistemi di misurazione personalizzati in funzione delle specificità della marca, degli obiettivi della campagna $\mathrm{e}$ del media mix utilizzato;

- indicatori tradizionali di risultato, come la customer satisfaction o il fatturato.

A fronte della condivisa rilevanza della misurazione, il quadro che emerge dalle interviste è di rapida evoluzione e di sperimentazione continua. L'unico dato abbastanza trasversale è l'utilizzo diffuso degli strumenti nativi digitali. Ma se le imprese, soprattutto pure play, fanno ampio ricorso a indicatori tradizionali (es. tasso di conversione, vendite), le agenzie sono più propense, invece, alle metriche ad hoc che vadano oltre le soluzioni standardizzate proposte dalle piattaforme social. Solo tra le imprese, alcuni lamentano l'incapacità non solo di impostare le misurazioni del CBE, ma anche di interpretare le metriche. 


\section{sinergie}

Vol. 36, N. 106, 2018

4.4 Le sfide manageriali del CBE (RQ4)

Per quel che concerne le sfide manageriali, dalle risposte degli intervistati sono emersi i seguenti sotto-temi (Fig. 5):

- branding;

- nuovi approcci di misurazione;

- integrazione online \& offline;

- superamento dellatteggiamento negativo verso il CBE.

Fig. 5: Le sfide manageriali del CBE: temi e sotto-temi

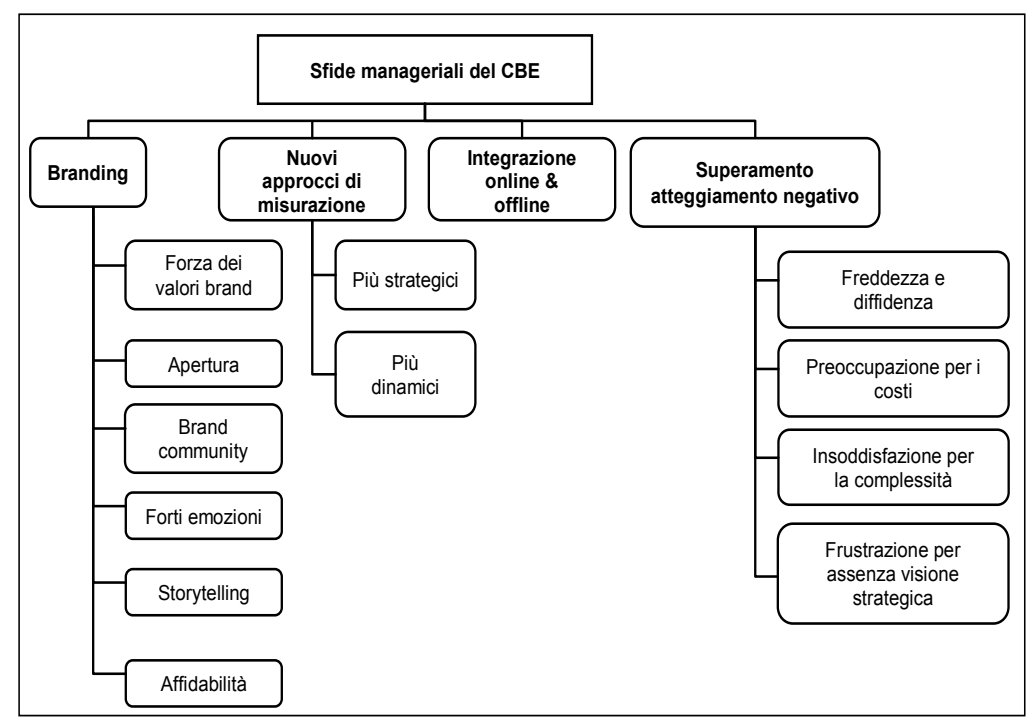

Fonte: nostra elaborazione

\section{Branding}

Le agenzie e le imprese hanno mostrato una visione molto ampia delle sfide per il branding, che possono essere declinate nei seguenti sotto-temi:

- rafforzamento dei valori identitari del brand, da leggere anche alla luce del significato di identificazione del CBE $(\$ 4.1)$ e degli asset della marca $(\$ 4.3)$;

- apertura del brand, che si traduce nella capacità della marca di ascoltare e di dialogare in modo trasparente con i consumatori e gli stakeholder rilevanti ( $\$ 4.1)$;

- capacità di sviluppare brand community, soprattutto online, facendo leva sull'engagement in termini di interattività sociale e di partecipazione $(\$$ $4.1)$;

- capacità di emozionare e appassionare, da intendersi strettamente correlata alla dimensione emozionale alla base del CBE emersa in precedenza $(\$ 4.1)$;

- capacità di storytelling, ossia di trasformare i valori fondanti del brand in storie da raccontare $(\$ 4.1)$; 
- mantenimento dell'affidabilità della marca nel tempo, grazie all'offerta di un prodotto di qualità che risponda costantemente a quanto richiesto dai consumatori. In tal modo, si può lavorare sulla creazione del brand trust, ritenuto particolarmente importante - soprattutto dalle imprese nella creazione di CBE $(\$ 4.2)$.

Lanalisi delle risposte per categoria mette in luce, anche in questo caso, alcune specificità. Per le agenzie, soprattutto digitali, risultano relativamente più importanti sfide quali l'apertura della marca, come pure la capacità di sviluppare brand community e un adeguato storytelling.

"Bisogna lasciare ai consumatori la possibilità di esprimere loro stessi $e$ di costruire la marca insieme allimpresa (la co-creation è un aspetto fondamentale nel tema dell'engagement). Si pensi ad esempio a Nutella, che dà al cliente la possibilità di personalizzare con i propri messaggi il prodotto, o a Nike, che offre l'opportunità di personalizzare la propria scarpa" (Account Director, Agenzia digitale).

Le agenzie tradizionali, invece, pur riconoscendo l'importanza dell'apertura, oltre che al focus sulla narrazione di marca, mostrano di dare priorità al rafforzamento dei valori identitari del brand.

Le imprese, dal canto loro, sembrano concentrarsi in particolare sulla forza dei valori e sull'affidabilità di prodotto/brand: come evidenziato sopra a proposito degli obiettivi del CBE $(\$ 4.2)$, questa categoria di attori risulta infatti relativamente più preoccupata dalla necessità di sviluppare la fiducia nel brand attraverso il mantenimento nel tempo delle promesse.

"Non basta solo raccontare delle belle storie, ma è la consistenza che fa la differenza e per la nostra azienda la consistency viene mostrata soprattutto tramite il servizio che offriamo e che garantiamo nel tempo" (Customer engagement \& Innovation Specialist, Impresa pure play).

\section{Nuovi approcci di misurazione}

Tale sfida è strettamente collegata alla necessità, evidenziata soprattutto dalle agenzie digital, di sviluppare un approccio che sia più strategico, ovvero in grado di identificare e di utilizzare le metriche più appropriate solo dopo aver strutturato un sistema di obiettivi e di KPI puntuali.

"La cosa fondamentale è stabilire prima quelli che sono $i \mathrm{KPI}$, ovvero $i$ valori sulla base dei quali valutare se l'engagement ha avuto successo oppure no... Il numero fine a se stesso non è interessante" (General Manager, Agenzia digitale).

Al tempo stesso, il nuovo approccio di misurazione dovrebbe essere più dinamico, per poter seguire l'evoluzione nel tempo del comportamento del consumatore, sempre più cross-mediale. Le imprese, in particolare quelle clicks-and-mortar, lamentano infine l'assenza di misure qualitative.

\section{Integrazione online e offline}

L'integrazione tra la comunicazione online e offline viene considerata da tutti i rispondenti come una sfida molto impegnativa e ancora ben lontana dall'essere superata.

"Andremo sempre più verso un universo di comunicazione in cui non ci sarà più bisogno di diversificare tra online e offline, ma sarà tutto interconnesso in maniera multi-piattaforma e multi-schermo: non si avrà più solo lo schermo televisivo o la piattaforma social poiché ambedue 
sinergie Vol. 36, N. 106, 2018

saranno sempre più integrati in una sorta di unico ecosistema che permetterà di interagire in maniera contemporanea" (Web Media Relations Manager, Impresa clicks-and-mortar).

Le imprese avvertono particolarmente tale sfida, focalizzando l'attenzione sugli ostacoli all'integrazione, che possono essere di tipo strategico, operativo e organizzativo. Con riferimento a quest'ultimo aspetto, nelle risposte delle imprese si rinviene una ancora non chiara collocazione delle persone preposte allo sviluppo del CBE, che possono trovarsi nella funzione marketing o in quella di comunicazione, nell'ufficio stampa/PR, nel customer service o, laddove presente, nella funzione digital.

"A livello formale, ad oggi, nella nostra azienda non esiste una funzione organizzativa che si occupa specificatamente della pianificazione e gestione dellengagement. Le dinamiche legate a questo tema vengono sostanzialmente affrontate dagli addetti alla comunicazione" (Global head of Performance Marketing, Impresa pure play).

"Esiste una funzione che gestisce la parte web e social ed è incaricata anche di misurare i risultati... Dal punto di vista dei contenuti che vengono condivisi, però, la matrice prevede ancora che la strategia nasca offline: l'azienda stabilisce quali sono i messaggi chiave e poi questi vengono declinati sul digitale e sugli altri canali" (Business Analyst, Impresa clicks-andmortar).

\section{Atteggiamento verso il CBE}

Dalle risposte degli intervistati emerge un relativo interesse di tutte le categorie verso il consumer-brand engagement, anche se sono diffusi trasversalmente atteggiamenti negativi, legati soprattutto alla difficoltà di capire le varie dimensioni dello stesso nei differenti touch point. Riguardo quindi agli atteggiamenti negativi che gli attori del communication network provano verso il CBE, i sotto-temi emersi dall'analisi del contenuto, con le specificità di cui diremo tra breve, sono i seguenti:

- freddezza/diffidenza verso il CBE, derivante dalla mancata comprensione delle effettive potenzialità dello stesso e, quindi, dalla sottovalutazione dei risultati che si potrebbero ottenere;

- preoccupazione per i costi elevati del CBE a fronte di risultati che tardano ad arrivare o che sono poco chiari;

- insoddisfazione verso il CBE a causa della complessità informativa/ di misurazione da gestire e alle criticità organizzative derivanti dall'adozione di una strategia orientata all'engagement;

- frustrazione, discendente dall'assenza di visione strategica che porta all'adozione di una logica di breve termine, orientata a ritorni immediati che non sono realisticamente raggiungibili.

Le differenze tra le categorie di rispondenti in questo caso appaiono molto evidenti. In particolare, le imprese sono più fredde e diffidenti verso il $\mathrm{CBE}$, ne sottovalutano la portata, sono preoccupate per i costi e, nell'insieme, sono insoddisfatte per la complessità - informativa, organizzativa e soprattutto culturale - da gestire.

"Secondo me, è una questione di complessità: fare brand engagement non è una passeggiata. Farlo significa per le aziende mettere in campo risorse professionali ad altissimo livello, quindi mettersi in discussione: ascoltare $i$ 
propri stakeholder è un atto di umiltà culturale e lo è, ancor più, quando si tratta di lasciarli entrare all'interno dei propri processi produttivi e decisionali (Web Media Relations Manager, Impresa clicks-and-mortar).

Le agenzie, soprattutto quelle digital, avvertono invece un senso di frustrazione nel dover supportare clienti ancora poco pronti a capire e apprezzare il CBE con una visione più ampia e strategica, essendo preoccupati principalmente dal contenimento dei costi e dai ritorni di breve periodo.

"Credo che si possa parlare di una difficoltà legata alla mancanza di una visione d'insieme della strategia e degli obiettivi da raggiungere da parte dell'impresa..." (CEO, Agenzia tradizionale).

"Le aziende [...] in questo momento di crisi preferiscono l'investimento in azioni che hanno un ritorno diretto sul business e, poi, la parte che "avanza" viene eventualmente investita in engagement ..." (Web Marketing Strategist, Agenzia digitale).

\section{Conclusioni}

I risultati della ricerca esplorativa fanno luce sui temi specifici considerati rilevanti da imprese e agenzie, che sono sempre più impegnate a collaborare nello sviluppo di strategie di CBE. Questi temi-chiave sono stati individuati induttivamente, approfondendo il punto di vista degli operatori con riferimento ai quattro nodi concettuali alla base del nostro framework, ossia: 1) i significati del CBE (RQ1); 2) gli obiettivi manageriali delle strategie di CBE (RQ2); 3) i sistemi gestionali del CBE (RQ3); e infine 4) le sfide manageriali del CBE (RQ4).

$\grave{E}$ emerso un quadro strategico assai articolato, dove a fianco di alcune prospettive condivise da tutti i diversi attori, vengono ad evidenza specificità di vedute non solo inter-categoria (agenzie versus imprese), ma anche intracategoria (clicks-and-mortar vs pure play e agenzie tradizionali vs agenzie digitali). Tali differenze sembrano derivare in primis dall'appartenenza a categorie differenti di attori del communication network (Vernuccio e Ceccotti, 2017), con ruoli e finalità di business diversi. Considerando poi la medesima categoria, le peculiarità dipendono spesso dal livello di maturità nellorientamento al digitale, riconducibile ad esempio al diverso livello di concentrazione del business nel mercato digitale (Chaffey et al., 2009) e al livello raggiunto nello sviluppo interno di competenze digitali (Vernuccio e Ceccotti, 2015). In grande sintesi, da tutti gli operatori il CBE è visto con grande attenzione e interesse, in particolare come: un concetto basato sulla relazione interattiva e dinamica con il consumatore; un obiettivo strategico di marketing e comunicazione, che è perseguibile soprattutto a partire dall'asset della marca grazie ai media digitali e all'integrazione tra online e offline, e per il quale ancora non sono state messe a punto soluzioni consolidate per la misurazione. Infine, sia imprese che agenzie hanno una visione piuttosto articolata delle sfide manageriali. Vi sono tuttavia anche notevoli specificità a seconda della categoria. Rispetto alle imprese, le agenzie dimostrano una maggiore capacità di lettura del fenomeno, con riferimento: al significato, di cui colgono pienamente le
Maria Vernuccio

Gabriele Qualizza

Andrea Buratti

Federica Ceccotti

Strategie di consumer-branc

engagement. Il punto di

vista delle imprese e delle agenzie di comunicazione 
sinergie Vol. 36, N. 106, 2018

molteplici sfaccettature; alle implicazioni manageriali in termini di varietà di obiettivi specifici, tra cui ad esempio la co-creazione; alle possibilità di elaborazione di metriche ad hoc; alle sfide più innovative come l'apertura del brand. La minore "maturità" manageriale delle imprese nell'affrontare le sfide poste dal CBE può essere ricondotta alla non adeguata conoscenza del fenomeno e delle sue implicazioni, alla sopravvalutazione dei risultati ottenibili nel breve termine, alla non corretta valutazione del rapporto risultato/investimento (percezione di costi elevati tout court) e all'incapacità di gestire la complessità gestionale e organizzativa.

Se prescindiamo dalla categoria, è interessante sottolineare alcune convergenze di vedute che sembrano essere influenzate soprattutto dal diverso livello di maturità digitale. Trasversale agli attori digitali (agenzie digitali e imprese pure play) è la logica consumer-centric, che ad esempio porta a valutare il cambiamento del consumatore come la causa principale della rilevanza del CBE. Tuttavia, in contraddizione con questa capacità di lettura del fenomeno, gli attori digitali mostrano un altro tipo di "miopia": sentendosi culturalmente e operativamente meno vincolati al mondo offline, sottovalutano di fatto l'importanza dell'integrazione tra offline e online.

In termini di implicazioni manageriali, la varietà di visioni emerse dalla ricerca ci porta a suggerire ai player del communication network un maggior dialogo, orientato alla comprensione reciproca sul tema del CBE a livello inter-categoria, in modo da sviluppare collaborazioni più efficaci.

Per le imprese, soprattutto pure play, possiamo sottolineare la necessità di riconciliare lenfasi sugli approcci tattici - largamente incoraggiati dallo sviluppo dei social media (che consentono di creare engagement attraverso azioni tattiche di real-time marketing) - con una visione di lungo termine, di respiro strategico. Le agenzie, d'altra parte, dovrebbero intensificare gli sforzi di educazione e formazione sul tema verso i propri clienti, al fine di superare le inadeguatezze da loro spesso lamentate nel lavoro di sviluppo di strategie di CBE. Inoltre, le agenzie non dovrebbero considerare solo i temi legati alla comunicazione, ma, ponendosi realmente al servizio dei propri clienti, dovrebbero dare un maggior peso a questioni strategiche per questi ultimi, come il brand trust e i risultati di marketing. Sia le imprese che le agenzie dovrebbero essere orientate al disegno di un approccio di misurazione dei risultati più strategico e più chiaramente e rigorosamente legato agli obiettivi prestabiliti e ai KPI.

Dal punto di vista accademico, il presente lavoro rappresenta la prima proposta di un framework concettuale che sia: 1) volto a integrare in ununica cornice i molteplici temi chiave di tipo manageriale affrontati nello sviluppo di strategie di CBE; 2) sviluppato induttivamente a partire dalladozione del punto di vista dei principali attori del communication network: da una parte, le imprese inserzioniste, clicks-and-mortar e pure play, e dallaltra, le agenzie di comunicazione, tradizionali e digitali. In tal modo, la nostra ricerca fornisce diversi contributi alla letteratura sullengagement. In primo luogo, il nostro contributo sposta il focus dell'attenzione degli studiosi del CBE dal punto di vista del consumatore, ampiamente indagato dal 2005 ad oggi (Islam e Rahman, 2016), a quello degli attori manageriali. In secondo luogo, considerando esclusivamente 
i rari studi precedenti focalizzati sulle imprese e/o sulle agenzie, il lavoro arricchisce lo stato dell'arte, proponendo un allargamento dell'area di indagine: la nostra ricerca, infatti, non si ferma agli aspetti definitori del CBE e alle strategie di branding (Gambetti et al., 2012; Vivek et al., 2012), ma analizza tutti i macro-temi di natura manageriale considerati più rilevanti dai professionisti coinvolti nello sviluppo di strategie di marketing e comunicazione. Un ulteriore valore aggiunto riguarda l'adozione inedita di una chiave di lettura manageriale più ampia rispetto al passato, in quanto va a considerare più punti di vista, che si palesano differenti in base al ruolo giocato dall'attore (impresa cliente $v s$ agenzia fornitrice) e/o in base al livello di orientamento al digital dello stesso (clicks-and-mortar vs pure play e agenzie tradizionali vs agenzie digitali). Ancora, il lavoro rappresenta un primo passo finalizzato a colmare il gap di analisi che si rileva con riferimento alle strategie di "orientamento all'engagement", le quali, seppure definite di recente a livello generale da Kumar e Pansari (2016), non sono state ancora approfondite empiricamente.

Considerando gli sviluppi futuri della ricerca, potrebbe essere interessante approfondire la prospettiva manageriale, adottando una visione specifica di settore o di dimensione d'impresa, comparando ad esempio le sfide manageriali percepite dalle grandi imprese con quelle di piccole e medie dimensioni. Anche il tema dello sviluppo del CBE per i brand locali apre un interessante ambito di investigazione che non è stato approfondito dalla letteratura precedente sul tema.

Infine, il presente lavoro non è esente da limitazioni, soprattutto legate alla natura qualitativa ed esplorativa dello studio che rende i risultati non generalizzabili. Da questo punto di vista, sono auspicabili studi anche di tipo quantitativo, in grado di ampliare l'orizzonte di analisi.

\section{Bibliografia}

AFUAH A., TUCCI C.L. (2001), Internet business models and strategies, McGrawHill, New York.

ANDERSON C. (2015), "Worldwide business consulting strategy for digital customer and brand engagement 2015 vendor assessment", www.idc.com

ARMSTRONG J.S. (1979), "Advocacy and objectivity in science", Management Science, vol. 25, n. 5, pp. 423-428.

BAZELEY P. (2007), Qualitative data analysis with NVivo, Sage Publications, London.

BLAU P. (1964), Exchange and power in social life, Wiley, New York.

BODINE K., HAGEN P., STONE A., MURPHY M. (2013), "The Customer Experience Ecosystem", Research report, Forrester Research. https:// www.forrester.com/report/The+Customer+Experience+Ecosystem/-/ERES59115 (ultimo accesso 12 novembre 2017).

BOYATZIS R.E. (1998), Transforming qualitative information: Thematic analysis and code development, Sage publication, Thousand Oaks, London.

BOWDEN J. (2009), "The process of customer engagement: a conceptual framework", Journal of Marketing Theory and Practice, vol. 17, n. 1, pp. 6374 .
Maria Vernuccio

Gabriele Qualizza

Andrea Buratti

Federica Ceccotti

Strategie di consumer-brand

engagement. Il punto di

vista delle imprese e delle agenzie di comunicazione 
BRAUN V., CLARKE V. (2006), "Using thematic analysis in psychology”, Qualitative Research in Psychology, vol. 3, n. 2, pp. 77-101.

BREIDBACH C.F., BRODIE R.J., HOLLEBEEK L. (2014), "Beyond virtuality: from engagement platforms to engagement ecosystems", Managing Service Quality, vol. 24, n. 6, pp. 592-611.

BRODIE R.J., HOLLEBEEK L.D., JURIĆ B., ILIĆ A. (2011), “Customer engagement: Conceptual domain, fundamental propositions, and implications for research", Journal of Service Research, vol. 14, n. 3, pp. 252-271.

BRODIE R.J., FEHRER J., JAAKKOLA E., HOLLEBEEK L. CONDUIT J. (2016), "From Customer to Actor Engagement: Exploring a Broadened Conceptual Domain", in Knoeferle K. (ed.), Marketing in the age of data, EMAC European Marketing Academy 2016 Conference, Oslo, May 24-27.

CABIDDU F., CARLO M.D., PICCOLI G. (2014), "Social media affordances: enabling customer engagement”, Annals of Tourism Research, vol. 48, September, pp. 175-192.

CALDER B.J., MALTHOUSE E.C., MASLOWSKA E. (2016a), "Brand marketing, big data and social innovation as future research directions for engagement", Journal of Marketing Management, vol. 32, n. 5-6, pp. 579-585.

CALDER B.J., ISAAC M.S., MALTHOUSE E.C. (2016b), "How to capture consumer experiences: a context-specific approach to measuring engagement: predicting consumer behavior across qualitatively different experiences", Journal of Advertising Research, vol. 56, n. 1, pp. 39-52.

CALDER B.J., MALTHOUSE E.C., SCHAEDEL U. (2009), "An experimental study of the relationship between online engagement and advertising effectiveness", Journal of Interactive Marketing, vol. 23, n. 4, pp. 321-331.

CALDER B.J., MALTHOUSE E.C. (2005), "Managing media and advertising change with integrated marketing", Journal of Advertising Research, vol. 45, n. 4, pp. 356-361.

CARDANO M. (2003), Tecniche di ricerca qualitativa, Carocci, Roma.

CARTER T. (2008), “Customer engagement and behavioral considerations”, Journal of Strategic Marketing, vol. 16, n. 1, pp. 21-26.

CECCOTTIF. (2018), Le agenzie di comunicazione nel nuovo scenario dell'Integrated Marketing Communication. Innovazione, competizione e relazioni, McGrawHill Education, Milano.

CHAFFEY D., ELLIS-CHADWICK F., MAYER R., JOHNSTON K. (2009), Internet marketing: strategy, implementation and practice, Financial Times Prentice Hall, Harlow.

CHANDLER J.D., LUSCH R.F. (2015), “Service Systems: A Broadened Framework and Research Agenda on Value Propositions, Engagement, and Service Experience", Journal of Service Research, vol. 18, n. 1, pp. 6-22.

CORBIN J., STRAUSS A. (2008), Basics of qualitative research, 3e, Sage, Los Angeles.

CRESWELL J.W. (1998), Qualitative inquiry and research design: choosing among five traditions, Sage Pubblications, Thousand Oaks, CA.

DELLI ZOTTI G. (2004), Introduzione dalla ricerca sociale, FrancoAngeli, Milano.

FOURNIER S. (1998), "Consumers and their brands: Developing relationship theory in consumer research", Journal of Consumer Research, vol. 24, n. 4, pp. 343-373.

GAMBETTI R.C., GRAFFIGNA G. (2010), "The concept of engagement: a systematic analysis of the ongoing marketing debate", International Journal of Market Research, vol. 52, n. 6, pp. 39-56. 
GAMBETTI R.C.C., GRAFFIGNA G., BIRAGHI S. (2012), “The grounded theory approach to consumer-brand engagement: The practitioner's standpoint”, International Journal of Market Research, vol. 54, n. 5, pp. 659-687.

GLASER B., STRAUSS A. (1967), The Discovery of Grounded Theory: Strategies for Qualitative Research, Aldine, Chicago.

GRAFFIGNA G., GAMBETTI R.C. (2015), "Grounding consumer-brand engagement: A field-driven conceptualisation”, International Journal of Market Research, vol. 57, n. 4, pp. 605-629.

GRANT I., MCLEOD C., SHAW E. (2012), "Conflict and advertising planning: Consequences of networking for advertising planning", European Journal of Marketing, vol. 46, n. 1/2, pp. 73-91.

HOLLEBEEK L. (2011), "Exploring customer brand engagement: Definition and themes", Journal of Strategic Marketing, vol. 19, n. 7, pp. 555-573.

HOLLEBEEK L.D., CONDUIT J., SWEENEY J., SOUTAR G., KARPEN I.O., JARVIS W., CHEN T. (2016a), "Epilogue to the Special Issue and reflections on the future of engagement research", Journal of Marketing Management, vol. 32, n. 5-6, pp. 586-594.

HOLLEBEEK L.D., GLYNN M.S., BRODIE R.J. (2014), "Consumer brand engagement in social media: Conceptualization, scale development and validation", Journal of Interactive Marketing, vol. 28, n. 2, pp. 149-165.

HOLLEBEEK L.D., SRIVASTAVA R.K., CHEN T. (2016b), "S-D logic-informed customer engagement: integrative framework, revised fundamental propositions, and application to CRM", Journal of the Academy of Marketing Science, first online: 06 september, pp. 1-25.

HOYER W.D., CHANDY R., DOROTIC M., KRAFFT M., SINGH S.S. (2010), "Consumer cocreation in new product development", Journal of Service Research, vol. 13, n. 3, pp. 283-296.

ISLAM J.U., RAHMAN Z. (2016), "The transpiring journey of customer engagement research in marketing: A systematic review of the past decade", Management Decision, vol. 54, n. 8, pp. 2008-2034.

JAAKKOLA E., ALEXANDER M. (2014), "The role of customer engagement behavior in value co-creation: a service system perspective", Journal of Service Research, vol. 17, n. 3, pp. 247-261.

KING N., HORROCKS C. (2010), Interviews in Qualitative Research, Sage Pubblications, Thousand Oaks, CA.

KOZINETS R. (2014), "Social brand engagement: A new idea", GfK Marketing Intelligence Review, vol. 6, n. 2, pp. 8-15.

KRIPPENDORFF K. (2004), Content analysis: An introduction to its methodology, SAGE Pubblications, Thousand Oaks, CA.

KUMAR V., PANSARI A. (2016), “Competitive advantage through engagement”, Journal of Marketing Research, vol. 53, n. 4, pp. 497-514.

LANGDRIDGE D. (2004). Introduction to Research Methods and Data Analysis in Psychology, Pearson Prentice Hall, Harlow.

LEMON K.N., VERHOEF P.C. (2016), "Understanding Customer Experience Throughout the Customer Journey", Journal of Marketing, vol. 80 (November), pp. 69-96.

LINCOLN Y.S., GUBA E.G. (1985), Naturalistic inquiry, SAGE Pubblications, Thousand Oaks, CA.
Maria Vernuccio

Gabriele Qualizza

Andrea Buratti

Federica Ceccotti

Strategie di consumer-brand

engagement. Il punto di

vista delle imprese e delle agenzie di comunicazione 
MASLOWSKA E., MALTHOUSE E.C., COLLINGER T. (2016), “The customer engagement ecosystem”, Journal of Marketing Management, vol. 32, n. 5-6, pp. 469-501.

MALTHOUSE E.C., CALDER B.J., TAMHANE A. (2007), "The Effects of media Context Experiences On Advertising Effectiveness", Journal of Advertising, vol. 36, n. 3, pp. 7-18.

MARKETING SCIENCE INSTITUTE (MSI) (2010), 2010-2012 Research priorities, Cambridge, MA.

MERSEY D.R., MALTHOUSE E.C., CALDER B.J. (2010), "Engagement with online media", Journal of media Business Studies, vol. 7, n. 2, pp. 39-56.

MOLLEN A., WILSON H. (2010), "Engagement, telepresence and interactivity in online consumer experience: Reconciling scholastic and managerial perspectives", Journal of Business Research, vol. 63, n. 9/10, pp. 919-925.

NEFF J. (2007), “OMD proves the power of engagement", Advertising Age, vol. 78, n. 27, pp. 3-4.

PANSARI A., KUMAR V. (2017), "Customer engagement: the construct, antecedents, and consequences", Journal of the Academy of Marketing Science, vol. 45, n. 3, pp. 294-311.

POWELL S. (2007), "Organisational marketing, identity and the creative brand", Journal of brand Management, vol. 15, n. 1, pp. 41-56.

REICHARDT C., COOK T. (1979), "Beyond Qualitative versus Quantitative Methods", in Cook T., Reichardt C. (eds), Qualitative and Quantitative Methods in Evaluation Research, Sage, London, pp. 7-30.

ROBSON S., FOSTER A. (1989), Qualitative Research in Action, Hodder and Stoughton, London.

SPROTT D., CZELLAR S., SPANGENBERG E. (2009), “The importance of a general measure of brand engagement on market behavior: Development and validation of a scale", Journal of Marketing Research, vol. 46, n. 1, pp. 92-104.

TAX S.S., MCCUTCHEON D., WILKINSON I.F. (2013), "The Service Delivery Network (SDN): A Customer-Centric Perspective of the Customer Journey", Journal of Service Research, vol. 16, n. 4, pp. 454-70.

THOMSEN S., STRAUBHAAR J., BOLYARD D. (1998), "Ethnomethodology and the study of online communities: exploring the cyber streets", Information Research, vol. 4, n. 1, pp. 4-11, http://informationr.net/ir/4-1/paper50.html (ultimo accesso 20 novembre 2017).

VAN DOORN J., LEMON K.N., MITTAL V., NASS S., PICK D., PIRNER P., VERHOEF P.C. (2010), "Customer engagement behavior: theoretical foundations and research directions", Journal of Service Research, vol. 13, n. 5, pp. 253-266.

VARGO S.L., LUSCH R.F. (2004), "Evolving to a new dominant logic for marketing”, Journal of Marketing, vol. 68, n. 1, pp. 1-17.

VARGO S.L., LUSCH R.F. (2008), "Service-dominant logic: continuing the evolution", Journal of the Academy of Marketing Science, vol. 36, n. 1, pp. $1-10$.

VARGO S.L., LUSCH R.F. (2016), "Institutions and axioms: an extension and update of service-dominant logic", Journal of the Academy of Marketing Science, vol. 44, n. 1, pp. 5-23.

VENKATESAN R. (2017), "Executing on a customer engagement strategy", Journal of the Academy of Marketing Science, vol. 45, n. 3, pp. 289-293. 
VERHOEF P.C., REINARTZ W.J., KRAFFT M. (2010), “Customer engagement as a new perspective in customer management", Journal of Service Research, vol. 13, n. 3, pp. 247-252.

VERNUCCIO M., CECCOTTI F. (2015), "Strategic and organisational challenges in the integrated marketing communication paradigm shift: A holistic vision", European Management Journal, vol. 33, n. 6, pp. 438-449.

VERNUCCIO M., CECCOTTI F. (2017), "Creative crowdsourcing e dinamiche relazionali nella comunicazione di marketing. Il caso del network di Zooppa”, Sinergie Italian Journal of Management, vol. 35, n. 103, pp. 57-80.

VIVEK S.D., BEATTY S.E., DALELA V., MORGAN R.M. (2014), "A generalized multidimensional scale for measuring customer engagement", Journal of Marketing Theory and Practice, vol. 22, n. 4, pp. 401-420.

VIVEK S.D., BEATTY S.E., MORGAN R.M. (2012), "Customer engagement: Exploring customer relationships beyond purchase", Journal of Marketing Theory and Practice, vol. 20, n. 2, pp. 122-146.

\section{Academic or professional position and contacts}

\section{Maria Vernuccio}

Associate Professor of Management

Sapienza University of Rome - Italy

e-mail: maria.vernuccio@uniroma1.it

\section{Gabriele Qualizza}

Research Fellow of Management

University of Udine - Italy

e-mail: gabriele.qualizza@uniud.it

\section{Andrea Buratti}

Post-doc Fellow of Management

University "Carlo Bo" of Urbino - Italy

e-mail: andrea.buratti@uniurb.it

\section{Federica Ceccotti}

Assistant Professor of Management Sapienza University of Rome - Italy

e-mail: federica.ceccotti@uniroma1.it

sinergie ISSN 0393-5108 DOI $10.7433 / \mathrm{s} 106.2018 .05$ pp. $81-107$ 